\title{
MiR-598 Suppresses Invasion and Migration by Negative Regulation of Derlin-1 and Epithelial-Mesenchymal Transition in Non-Small Cell Lung Cancer
}

\author{
Fengming Yanga,b Ke Weic Zhiqiang Qin ${ }^{\mathrm{d}}$ Weitao Liu ${ }^{\mathrm{e}}$ Chuchu Shao ${ }^{\mathrm{b}}$ \\ Chaoshan Wang ${ }^{\mathrm{e}}$ Ling Ma ${ }^{\mathrm{b}}$ Mengyan Xie ${ }^{b}$ Yongqian Shu ${ }^{\mathrm{a}, \mathrm{b}}$ Hua Shen ${ }^{\mathrm{a}, \mathrm{b}}$
}

\begin{abstract}
aDepartment of Oncology, The Affiliated Sir Run Run Hospital of Nanjing Medical University, Nanjing, bDepartment of Oncology, The First Affiliated Hospital of Nanjing Medical University, Nanjing, 'Department of Thoracic and Cardiovascular Surgery, The First Affiliated Hospital of Nanjing Medical University, Nanjing, dDepartment of Urology, The First Affiliated Hospital of Nanjing Medical University, Nanjing, 'Department of Pathology, Nanjing Medical University, Nanjing, China
\end{abstract}

\section{Key Words}

MicroRNA-598 • Derlin-1 • Non-small cell lung cancer $\bullet$ EMT $・$ Migration $・$ Invasion

\begin{abstract}
Background/Aims: MicroRNAs regulate a wide range of biological processes of non-small cell lung cancer (NSCLC). Although miR-598 has been reported to act as a suppressor in osteosarcoma and colorectal cancer, the physiological function of miR-598 in NSCLC remains unknown. In this study, the role of miR-598 in NSCLC was investigated. Methods: Quantitative real-time polymerase chain reaction (qRT-PCR) was conducted to estimate the expression of miR-598 and Derlin-1 (DERL1) in both NSCLC tissues and cell lines. Immunohistochemistry (IHC) analyzed the association between the miR-598 expression and epithelial-mesenchymal transition (EMT) hallmark genes (E-cadherin, Vimentin) by staining the tumors representative of the high- and low-expression groups. The effect of miR-598 and DERL1 on invasion and migration was determined in vitro using transwell and wound-healing assays. The molecular mechanism underlying the relevance between miR-598 and DERL1 was elucidated by luciferase assay and Western blot. Western blot assessed the expression levels of EMT hallmark genes in cell lines. Xenograft tumor formation assay was conducted as an in vivo experiment. Results: In this study, a relatively low level of miR-598 and high DERL1 expressions were found in NSCLC specimens and cell lines. IHC results established a positive correlation between the miR-598 expression and E-cadherin and a negative with Vimentin. DERL1 was verified as a direct target of miR-598 by luciferase assay. In vitro, the over-expression of miR-598 negatively regulated DERL1 and EMT for the suppression of invasion and migration. In vivo, the over-expression of miR-598 could inhibit tumor cell metastasis in NSCLC. Conclusions: These findings for the first time revealed that miR-598, as a tumor suppressor, negatively regulate DERL1 and EMT to suppress the invasion and migration in NSCLC, thereby putatively serving as a novel therapeutic target for NSCLC clinical treatment.

F. Yang, K. Wei, Z. Qin and W. Liu contributed equally to this work.




\section{Introduction}

Lung cancer is a frequent cause of cancer-related mortality worldwide with nearly 2.2 million new cases and 1.5 million deaths each year [1, 2]. Based on diverse biological characteristics and therapies, it can be categorized as small cell lung cancer (SCLC) and non-small cell lung cancer (NSCLC) [3]. The latter is the most common type of lung cancer (accounts for approximately 80\%) and is subdivided into squamous cell carcinoma, adenocarcinoma, and large cell carcinoma [4]. Despite the considerable advances in cancer surgery, chemotherapy, and molecular targeting therapies, the outcome of lung cancer patients remains poor. Invasion and migration of tumor cells are major challenges in the clinical treatment of NSCLC [5]. Consequently, the identification of novel molecules that inhibit the invasion and migration of lung cancer cells is imminent.

MicroRNAs (miRNAs) refer to a class of evolutionary conserved small noncoding RNA molecules containing 18-25 nucleotides that are critical post-transcriptional regulators of gene expression via binding to the 3'-untranslated region (3'-UTR) of target mRNAs [6]. A wide range of biological processes including cell proliferation, apoptosis, invasion, and migration have been identified to be regulated by miRNAs $[7,8]$. Thus, the deregulated expression of miRNAs has been associated with various diseases including cancers [9-12]. MiRNAs can be regarded as oncogenes or tumor suppressor genes based on their multiple target mRNAs. Recently, Liu et al. reported that miR-598 acts as a tumor suppressor in the proliferation, migration, and invasion of osteosarcoma cells [13]. Another study by Chen et al. revealed that the over-expression of miR-598 suppresses the metastasis in colorectal cancer by targeting JAG1 [14]. Nonetheless, the role of miR-598 in NSCLC has not yet been reported.

In the present study, we identified miR-598 as a tumor suppressor, which effectuates by direct binding to the 3'-UTR of Derlin-1 (DERL1) in NSCLC cell lines. The family of Der1 proteins has emerged as a vital component of the ERAD pathway [15]. DERL1 is a partner of the p97 ATPase complex and plays a key role in the mislocalization of the misfolded protein; it also integrates into the endoplasmic reticulum (ER) membrane to survey such protein aggregates $[16,17]$. Recently, several studies have demonstrated that DERL1 contributes to carcinogenesis and tumor progression, and the upregulation of DERL1 expression promotes the development of colon cancer, esophageal cancer, and bladder cancer [18-20]. In addition, DERL1 is consistently overexpressed in NSCLC and correlated with tumor grade and lymph node metastasis. Furthermore, it promotes the invasion by the upregulation of matrix metalloproteases (MMP) [21]. The expression of DERL1 in lung cancer was altered by ER stress. The knockdown of DERL1 usually impairs the degradation of p62 under ER stress [22]. However, the correlation between miR-598 and DERL1 has not yet been elucidated.

In summary, for the first time, we demonstrated that miR-598 inhibits the invasion and invasion by directly targeting DERL1. Also, the expression of epithelial-mesenchymal transition (EMT) hallmark genes was altered after upregulation or downregulation of the expression of miR-598 in NSCLC cells. Taken together, we suggested that miR-598 suppresses the invasion and migration by negative regulation of DERL1 and EMT in NSCLC.

\section{Materials and Methods}

\section{Clinical samples}

Sixty pairs of NSCLC and adjacent normal tissues were collected from patients diagnosed with NSCLC at the First Affiliated Hospital of Nanjing Medical University (Jiangsu, China) between 2012 and 2015. Each pair of NSCLC and adjacent tissue was obtained from the same patient after pathological examination, transferred into liquid nitrogen immediately, and stored at $-80{ }^{\circ} \mathrm{C}$ before use. This study was approved by the Ethics Committee of the First Affiliated Hospital of Nanjing Medical University.

Cell lines and Cell culture

Human NSCLC cell lines (A549, H1299, PC-9, H358, SPC-A1) were purchased from the Institute of Biochemistry and Cell Biology at the Chinese Academy of Sciences (Shanghai, China), and a normal human 


\section{Cellular Physiology Cell Physiol Biochem 2018;47:245-256

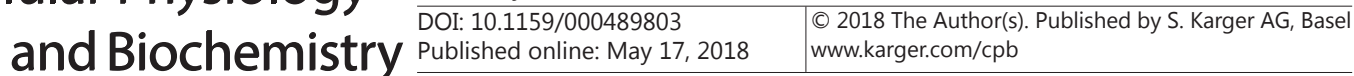

Yang et al.: Mir-598 in NSCLC

bronchial epithelial cell line (BEAS-2B) was purchased from ATCC (Manassas, VA, USA). All cell lines were cultured in RPMI 1640 or DMEM (GIBCO-BRL) supplemented with $10 \%$ fetal bovine serum (FBS), 100 U/ $\mathrm{mL}$ penicillin, and $100 \mathrm{mg} / \mathrm{mL}$ streptomycin. The cells were cultured in a humidified atmosphere at $37{ }^{\circ} \mathrm{C}$ with $5 \% \mathrm{CO}_{2}$.

\section{Construction of stable cell lines and cell transfection}

Human lung cancer cells, H1299 and SPCA1, were transfected with the lentiviral vectors that were used to construct LV-hsa-miR-598-mimic vector (miR-598) and the LV-hsa-miR-598-inhibitor vector (antimiR-598) (GenePharma, Shanghai, China). The LV empty lentiviral construct (NC and anti-NC) served as a negative control. The cells were infected with lentiviruses and screened under the selection pressure of puromycin according to the standard protocols. For overexpression of the target, the human DERL1 was cloned into pcDNA3.1-DERL1 (GenePharma). For the downregulation of DERL1 expression, siRNA targeting the encoding region of DERL1 was purchased from GenePharma, and the siRNA transfection reagent (Invitrogen, USA) was utilized following the manufacturer's instructions. The non-targeting control siRNA was used as a negative control.

\section{Isolation of total RNA and quantitative real-time polymerase chain reaction ( $q R T-P C R$ )}

Total RNAs were extracted from selected cell lines or tumor tissues using TRIzol reagent (Takara, Japan) according to the manufacturer's protocols. MiR-598 and DERL1 mRNA levels were quantified by qRT-PCR by miRNA UPL probe assay using Roche UPL probe \#21 (Haoqin, Shanghai, China) on ABI 7900 fast Real-time PCR system (ABI, CA, USA).

\section{Transwell assay}

Transwell chamber inserts with uncoated Matrigel (migration) and coated Matrigel (invasion) were used for cell migration and invasion assays, respectively. The transfected cells $\left(6 \times 10^{3}\right.$ cells $)$ were seeded into the upper chamber of the 24-well culture inserts coated with $200 \mu \mathrm{L}$ serum-free medium. $400 \mu \mathrm{L}$ complete medium supplemented with $10 \%$ FBS was added to the bottom of the inserts, allowing cells to migrate for $36 \mathrm{~h}$ and invade for $48 \mathrm{~h}$. After incubation, the cells on the upper surface of the membrane were removed, whereas those on the lower filter surfaces were fixed and stained with crystal violet. The number of migrated cells was counted under a microscope.

\section{Wound-healing assay}

For cell motility assay, the transfected cells were seeded in 6-well plates and cultured to almost confluency. A single wound was created by scratching the confluent monolayer of the cell using a $20-\mu \mathrm{L}$ sterile pipette tip. The cells were washed two times with phosphate-buffered saline (PBS) and incubated with DMEM supplemented with 1\% FBS. Images were taken at $0 \mathrm{~h}$ and $48 \mathrm{~h}$ after wounding.

\section{Bioinformatics analysis}

The potential targets of miR-598 were predicted and analyzed bioinformatically. In this study, we utilized four publicly available algorithms including PicTar, TargetScan, MiRWalk, and MiRanda. The results indicated that 3'-UTR of DERL1 binds to miR-598 providing a high score, suggesting that DERL1 might be a potential downstream target of miR-598.

\section{Luciferase reporter assay}

Human DERL1 3'-UTR sequence or the mutant sequence of DERL1 3'-UTR containing the predicted binding sites of miRNA-598 was inserted into pGL3 promoter vector (Genscript, Nanjing, China). Cell line H1299 and SPCA1 were seeded in 24-well plates $\left(5 \times 10^{5}\right.$ cells/well) on the day before transfection. Subsequently, the cells were co-transfected with luciferase reporter vectors $(0.12 \mu \mathrm{g})$ and miR-598 mimic or negative control using Lipofectamine 3000 Reagent (Invitrogen, USA). The luciferase reporter assay was conducted $48 \mathrm{~h}$ after transfection according to the manufacturer's instructions.

\section{Western blotting}

Cells were collected and lysed in RIPA lysis buffer (Beyotime, Shanghai, China). Total protein lysates extracted from tissues or cells were subjected to $10 \%$ sodium dodecyl sulfate-polyacrylamide gel 


\begin{tabular}{|c|c|c|}
\hline Cellular Physiology & Cell Physiol Biochem 2018;4 & 7:245-256 \\
\hline and Biochemistry & $\begin{array}{l}\text { DoI: 10.1159/000489803 } \\
\text { Published online: May 11, } 2018\end{array}$ & $\begin{array}{l}\text { O } 2018 \text { The Author(s). Published by S. Karger } A G \text {, Base } \\
\text { www.karger.com/cpb }\end{array}$ \\
\hline
\end{tabular}

electrophoresis (SDS-PAGE) and transferred to polyvinylidene fluoride (PVDF) membrane. The membrane was blocked, incubated with 5\% non-fat milk in mixture of Tris-buffered saline (TBS) containing Tween 20 (TBST) for $2 \mathrm{~h}$ at room temperature, and incubated with primary antibody against DERL1 (Abcam, USA), E-cadherin (Proteintech, USA), N-cadherin (Proteintech), Vimentin (Cell Signaling Technology; CST, USA), Snail (CST), GAPDH (CST), and $\beta$-actin (Abcam) at $4{ }^{\circ} \mathrm{C}$ overnight. The densitometric analysis of the immunoreactive bands was performed using Image J software.
Table 1. Expression levels of miR-598 and DERL1 in NSCLC tissues. NSCLC, non-small cell lung cancer; AD, adenocarcinoma; SCC, squamous cell carcinoma, *indicates $\mathrm{P}<0.05$ (Chi-quare test)

\begin{tabular}{lccccccc}
\hline \multirow{2}{*}{ Factors } & \multirow{2}{*}{ Number } & miR-598 & expresion & \multicolumn{5}{c}{ DERL1 expression } & \multirow{2}{*}{ Low } & High & P-Value & Low & High & P-Value \\
\hline Gender & & & & 0.88 & & & 0.6 \\
Male & 26 & 12 & 14 & & 14 & 12 & \\
Female & 34 & 15 & 19 & & 16 & 18 & \\
Age(years) & & & & 0.60 & & & 0.77 \\
$<60$ & 29 & 16 & 13 & & 17 & 12 & \\
$\geq 60$ & 31 & 15 & 16 & & 17 & 14 & \\
Smoking status & & & & 0.45 & & & 0.60 \\
Non-smoker & 28 & 13 & 15 & & 13 & 15 & \\
Smoker & 32 & 18 & 14 & & 17 & 15 & \\
Histology & & & & 0.87 & & & 0.71 \\
SCC & 27 & 12 & 15 & & 11 & 16 & \\
AD & 33 & 14 & 19 & & 15 & 18 & \\
Lymph node & & & & $0.017^{*}$ & & & $0.035^{*}$ \\
No metasis & 15 & 4 & 11 & & 10 & 5 & \\
Metasis & 45 & 28 & 17 & & 16 & 29 & \\
TNM stage & & & & $0.021^{*}$ & & & $0.037^{*}$ \\
I+II & 34 & 12 & 22 & & 21 & 13 & \\
III+IV & 26 & 17 & 9 & & 9 & 17 & \\
\hline
\end{tabular}

Immunohistochemistry (IHC) and H\&E staining

The expression of E-cadherin and Vimentin was examined by IHC staining. Briefly, the tissues were sliced into 3- $\mu \mathrm{m}$ sections and deparaffinized. Subsequently, the sections were incubated with antibodies against E-cadherin (Proteintech) or Vimentin (CST) at $4{ }^{\circ} \mathrm{C}$ overnight. After washing three times with PBS, all sections were incubated with goat anti-rabbit IgG for $30 \mathrm{~min}$ and stained with the color reagent 3, 3'-diaminobenzidine (DAB). Hematoxylin and eosin (H\&E) staining was utilized to visualize the metastatic nodules in the lungs of mice.

\section{In vivo metastasis analysis}

The Animal Care and Use Committee approved the animal experiments that were carried out in accordance with the institutional guidelines. BALB/c nude mice, $\geq 5$-week-old, were purchased from the Animal Center of Nanjing University. For the tail vein xenograft, 5 mice in each group were injected with $1 \times 10^{7}$ cells suspended in $100 \mu \mathrm{L}$ PBS through the tail vein. All the mice were sacrificed after 6 weeks postinoculation. Consequently, the metastatic nodules in the lungs were examined by necropsy and counted. The metastatic node was monitored by the IVIS@ Lumina II system every 5 days. Then, the lung tissues were fixed in 10\% neutral PB-buffered formalin. The fixed samples were embedded in paraffin and stained using IHC and H\&E as described above.

\section{Statistical analysis}

All experiments were performed in triplicate independently, and the chi-squared test was used to analyze the significant differences between the data shown in Table 1. Student's unpaired t-test was adopted to test the significant differences in other results, and the data were presented as mean \pm standard deviation (SD). All statistical analyses were performed using SPSS 19.0 software and $\mathrm{P}<0.05$ was considered as statistically significant.

\section{Results}

MiR-598 expression is downregulated in NSCLC

To investigate the role of miR-598 in NSCLC, we first detected the expression of miR-598 in 60 pairs of paired tumor tissues and adjacent normal tissues. The results showed that 
miR-598 was significantly downregulated in tumor tissues as compared to the adjacent noncancerous tissues (Fig. 1A). The expression levels of miR-598 in five NSCLC cell lines and the normal lung cell line (BEAS-2B) were analyzed by qRT-PCR. As shown in Fig. 1B, all five NSCLC cells showed a significant downregulation of miR-598 as compared to the normal lung cell line BEAS-2B. Reportedly, A549 and H1299 are highly metastatic cell lines, while SPCA1 and H358 are weak metastatic cell lines [23]. The expression level of miR-598 was markedly suppressed in highly metastatic cells (A549, H1299) as compared to that in weak metastatic cells (SPCA1, H358). This result also confirmed that the miR-598 expression was correlated with the metastatic ability of NSCLC cells. Subsequently, the chi-square test was used to study the relationship between miR-598 level and clinicopathological features of NSCLC in 60 patients. The expression of miR-598 was negatively correlated with lymph node metastasis $(\mathrm{P}=0.017)$ and TNM stage $(\mathrm{P}=0.029)$. However, no significant correlation was observed between the expression of miR-598 and other clinicopathological parameters (Table 1). An analysis of the association between miR-598 and EMT hallmark genes (E-cadherin, Vimentin) was performed by staining the tumors representative of high- and low-expression groups. The results showed a positive correlation between the miR-598 expression and E-cadherin and a negative correlation between miR-598 expression and Vimentin (Fig. 1C, D). These data suggested that miR-598 may exert an inhibitory role in NSCLC.

\section{MiR-598 inhibits invasion and migration of NSCLC cells}

To further reveal the role of miR-598 in NSCLC, we selected H1299 cells (with a relatively low expression of miR-598) and SPCA1 cells (with a relatively high expression of miR-598) for subsequent research. H1299 and SPCA1 cells were transfected with lentiviral constructs designed to overexpress or knockdown miR-598 (H1299-LV-miR-598, H1299LV-NC, SPCA1-ANTI-miR-598, and SPCA1-ANTI-NC). The transfection efficiency of the cell lines was validated via qRT-PCR. The results showed that the expression of miR-598 was increased in $\mathrm{H} 1299$ cells when transfected with miR-598 mimics, whereas miR-598 level was decreased in SPCA1 cells after transfection with anti-miR-598 (Fig. 2A). The transwell and wound healing assays investigated the migration and invasion of NSCLC. The migration and invasion ability of H1299 cells (miR-598 overexpressed group) could be suppressed by miR598. Conversely, the miR-598 inhibition group resulted in a significantly increased tendency of cell migration and invasion (Fig. 2B, C), thereby suggesting that miR-598 inhibited the invasion and migration of NSCLC cells.

Fig. 1. Expression of miR598 in NSCLC clinical samples and cell lines. A: The expression levels of miR-598 in 60 pairs of human NSCLC tissues and non-NSCLC tissues were explored using miRNA RT-PCR. B: The expression levels of miR-598 in five lung cancer cell lines (H1299, A549, PC-9, H358, SPC-A1) and normal lung cell (BEAS-2B). C, D: IHC staining against EMT hallmark genes (E-cadherin, Vimentin) collected from human NSCLC tissues showed a positive correlation between the miR-598 expression and E-cadherin and a negative correlation between the miR-598 expression and Vimentin. Data are represented as mean $\pm \mathrm{SD}$. *indicates $\mathrm{P}<0.05$, ${ }^{* *} \mathrm{P}<0.01$.

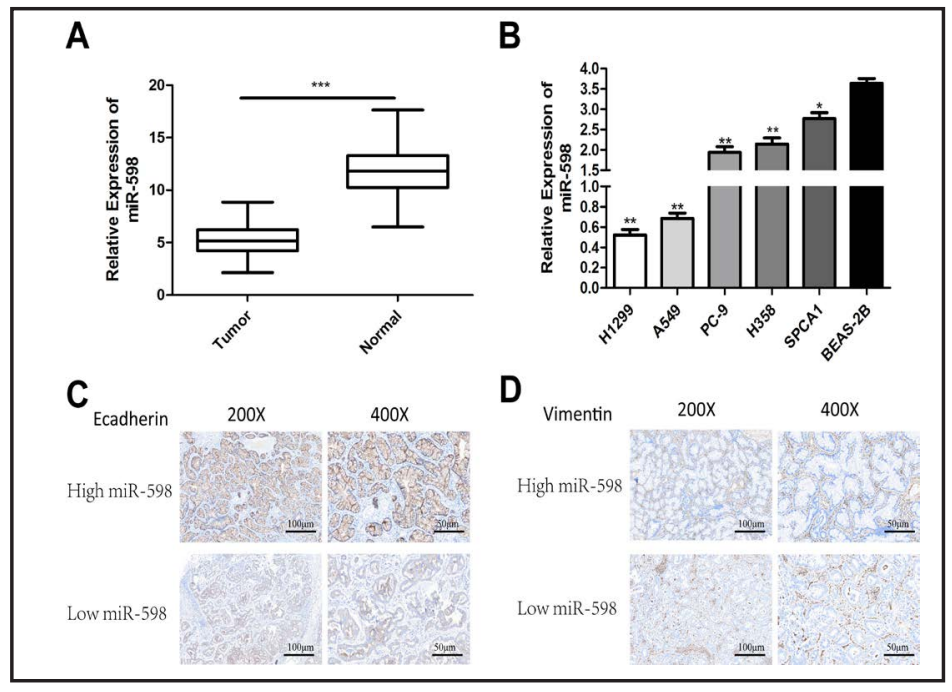


MiR-598 regulates
DERL1 expression by
directly binding to $3^{\prime}-$
UTR

To clarify the molecular mechanisms of miR598 in mediating NSCLC invasion and migration, four publicly available algorithms including PicTar, TargetScan, MiRWalk, and MiRanda were used to identify DERL1 as the putative target of miR598. This prediction was confirmed by a dualluciferase reporter system, where miR-598 was cotransfected with luciferase reporter plasmids containing the 3'-UTR of DERL1 or mutated DERL1 (harboring deletions of the putative miR-598 target sites). As shown in Fig. 3A, the co-transfection of miR598 mimics suppressed the luciferase activity of the reporter (containing the wild-type DERL1-UTR sequence). In addition, miR-598 mimics did not affect the luciferase activity when the target cells were transfected with mutated DERL1. These results suggested that DERL1 might be a direct functional target of miR-598 in NSCLC. The above results demonstrated that miR-598 level was expressed at a low level in NSCLC cancer tissues and cell lines (Fig. 1A, B). Next, we detected the expression level of DERL1 in 60 paired tumor tissues and the adjacent non-cancerous tissues by qRT-PCR and found that DERL1 was markedly upregulated in tumor tissues as compared to adjacent non-cancerous tissues (Fig. 3B). Furthermore, a two-tailed Pearson's correlation analysis evaluated the correlation between miR-598 and DERL1and found that the expression of miR-598 was negatively correlated with DERL1 expression (Fig. 3C). Also, the expression of DERL1 was correlated with lymph node metastasis $(\mathrm{P}=0.035)$ and $\mathrm{TNM}$ stage $(\mathrm{P}=0.041)$. However, no significant correlation was observed between the expression of DERL1 and other clinicopathological parameters (Table 1). In order to verify the role of DERL1 in NSCLC, the expression level of DERL1 was analyzed in NSCLC cell lines. Consequently, a significantly high DERL1 expression was observed in NSCLC cell lines (H1299, A549, PC9, H358, and SPCA1) as compared to the corresponding expression levels in normal lung cells (BEAS-2B) (Fig. 3D). These results indicated that DERL1 might act as a carcinogenic gene in NSCLC cells. Subsequently, the mRNA and protein levels of DERL1 were found to be reduced as a result of transfection with 
Fig. 3. MiR-598 regulates DERL1 expression by directly binding its 3'-UTR. A: Luciferase reporter assay was conducted to verify that miR-598 directly interacted with the 3'-UTR region of DERL1. Luciferase activity was analyzed in cells co-transfected with miR-598-mimics or negative control with pGL3DERL1-WT or pGL3-DERL1-MUT. B: The mRNA expression level of DERL1 relative to GAPDH in human NSCLC tissues and corresponding adjacent tissues was detected by qRT-PCR. C: Expression levels of DERL1 in five lung cancer cell lines (H1299, A549, PC-9, H358, SPCA1) and normal lung cell (BEAS2B). D: A negative correlation was observed between miR-598 and DERL1 in NSCLC samples. E: The protein expression and mRNA expression levels of DERL1 in transfected SPC-A1 and H1299 cells were analyzed by Western blot and qRT-PCR. $\beta$-actin served as an endogenous control. Data are represented as mean $\pm \mathrm{SD}$. *indicates $\mathrm{P}<0.05,{ }^{* *} \mathrm{P}<0.01$.

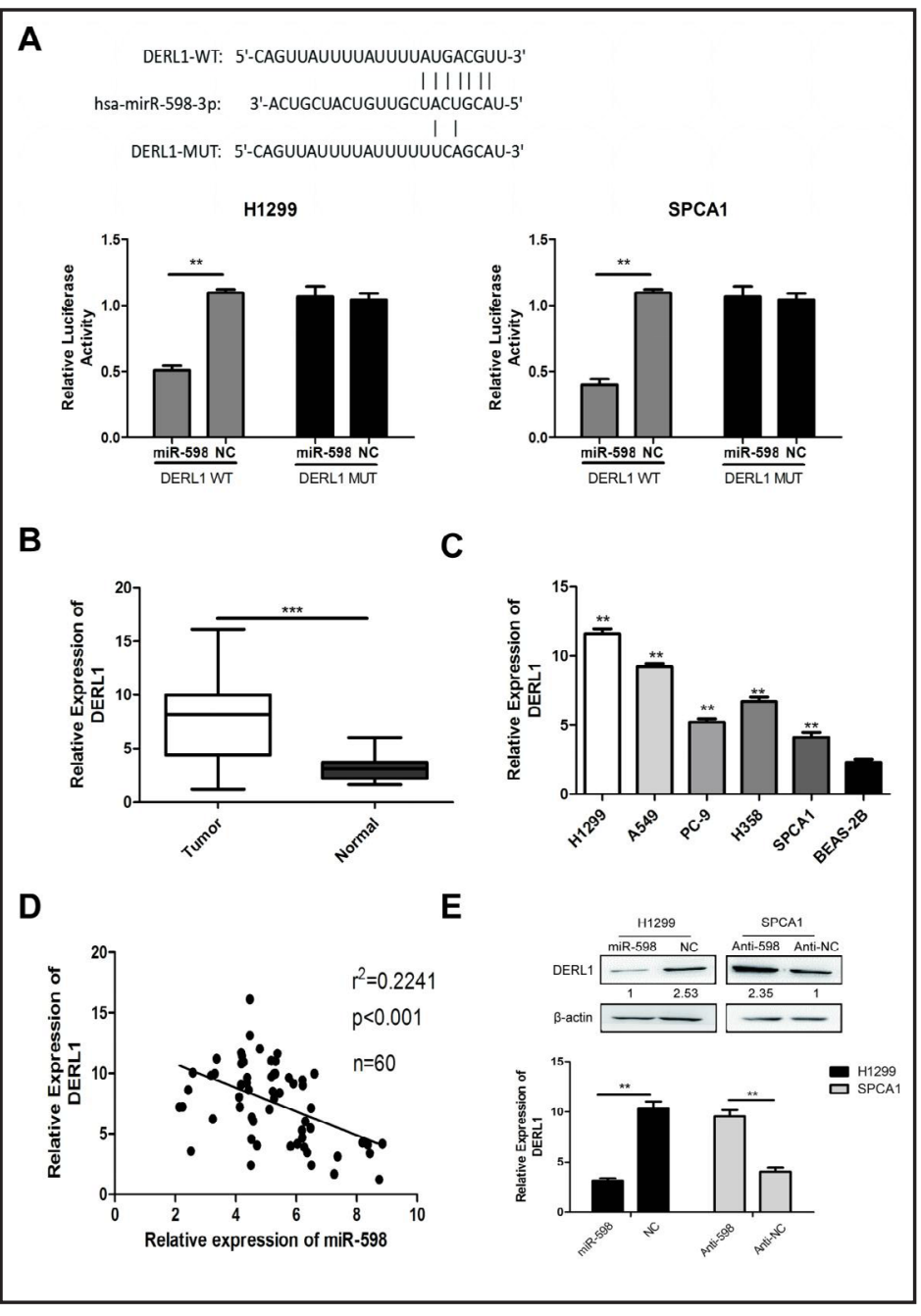

miR-598 mimics. Conversely, the expression of DERL1 was increased after transfection with miR-598 inhibitor (Fig. 3E, F). Collectively, we demonstrated that the oncogene DERL1 is a direct target gene of miR-598 and the expression level inhibited by miR-598.

MiR-598 suppresses invasion and migration of NSCLC cells by targeting DERL1

To elucidate whether the invasion and migration induced by aberrant expression of miR-598 were associated with the expression of DERL1 in NSCLC cells, we conducted rescue assays. The rescue experiment for miR-598 overexpression was performed by ectopic expression of DERL1 without its 3'-UTR in H1299 cells. Moreover, a similar rescue experiment for miR-598 silencing was performed by the downregulation of DERL1 in SPCA1 cells. The expression of DERL1 at both mRNA and protein levels in co-transfected cells was examined by qRT-PCR and Western blot analysis, respectively (Fig. 4A, B). In comparison with the control group, the upregulated expression of miR-598 in H1299 cells co-transfected with DERL1 without its 3'-UTR exhibited an increased migration and invasion ability. Consistently, the downregulated expression of miR-598 in SPCA1 cells co-transfected with si-DERL1 exhibited a decreased migration and invasion ability (Fig. 4C, D).

MiR-598 regulates the expression of EMT hallmark genes

EMT is known as a critical process in tumor invasion and migration. To gain further insight into the effects of miR-598 on EMT, Western blot was carried out to assess the expression levels of EMT regulatory proteins in cell lines. Consequently, the expressions of EMT hallmark 
proteins (E-cadherin, $\mathrm{N}$-cadherin, Vimentin, and Snail) were altered related to miR-598 expression as by Western blot (Fig. 5A). Interestingly, when miR-598 was overexpressed in H1299 cells, the level of epithelial marker E-cadherin was increased, while that of the mesenchymal markers, $\mathrm{N}$-cadherin, Vimentin, and Snail, was decreased as demonstrated by Western blot. These results indicated that the overexpression of miR-598 might suppress the EMT in NSCLC. On the other hand, E-cadherin was markedly suppressed in response to a miR-598 downregulation in SPCA1 cells, whereas N-cadherin, Vimentin, and Snail were induced, which suggested that the downregulated expression of miR-598 stimulated the EMT process. Moreover, the changes in the EMT markers were reversed in response to co-transfection with miR598 mimics and pcDNA3.1DERL1 (Fig. 5B) in NSCLC cells. Thus, we hypothesized that the overexpression of miR-598 could inhibit the migration and invasion potentially via suppressing EMT in NSCLC. Taken together, these findings suggested that miR-598 suppresses the invasion and migration by negatively regulating DERL1 and EMT.

MiR-598 inhibits NSCLC metastasis in vivo

To further assess the effect of miR-598 on the invasion and migration of NSCLC cell lines in vivo, we established a xenograft model utilizing the H1299 cells overexpressing miR-598 and SPCA1 cells with knocked down miR-598 via tail vein injection into nude mice. The metastatic node was measured by the IVIS@ Lumina II system. As shown in Fig. 6A, the miR-598-overexpressed mice presented less metastatic nodes than the control group, while mice with inhibited miR-

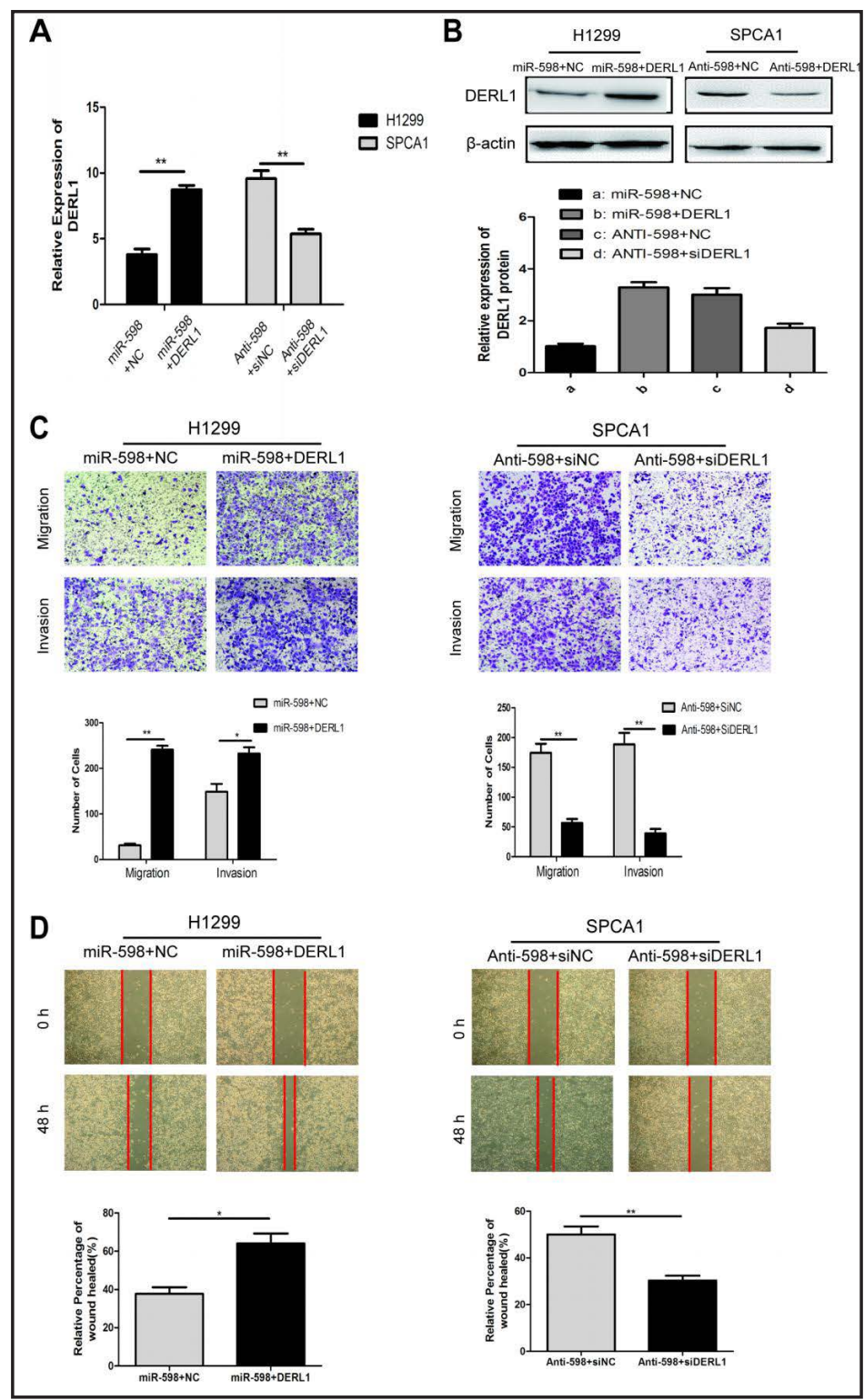

Fig. 4. MiR-598 suppresses invasion and migration of NSCLC cells by targeting DERL1. A: The expression of DERL1 was verified by qRTPCR in cotransfected cell lines. B: Western blot verified the expression of DERL1. C, D: The roles of miR-598 and DERL1 in the regulation of NSCLC cell migration and invasion were examined by transwell assay and wound-healing assay. The rescue experiment for miR-598 overexpression was performed by ectopic expression of DERL1 without the 3'-UTR in H1299 cells. A similar rescue experiment for miR-598 silencing was performed by downregulation of DERL1 in SPC-A1 cells. Data are represented as mean $\pm \mathrm{SD}$. *indicates $\mathrm{P}<0.05$, ${ }^{* *} \mathrm{P}<0.01$. 


\begin{tabular}{|c|c|c|}
\hline Cellular Physiology & Cell Physiol Biochem 2018; & $77: 245-256$ \\
\hline and Biochemistry & $\begin{array}{l}\text { DOI: 10.11159/000489803 } \\
\text { Published online: May 11, } 2018\end{array}$ & $\begin{array}{l}\text { O } 2018 \text { The Author(s). Published by S. Karger AG, Basel } \\
\text { www.karger.com/cpb }\end{array}$ \\
\hline
\end{tabular}

Fig. 5. MiR-598 regulates the expression of EMT hallmark genes. A: Expression levels of E-cadherin, N-cadherin, Vimentin, and Snail proteins in $\mathrm{H} 1299$ were determined after transfecting miR-598 mimics and miR-598-inhibitor, respectively in comparison with the control. B. The rescue experiments for miR-598 overexpression were performed by ectopic expression of DERL1 without the 3'UTR, and those for miR-598 silencing were performed by downregulation of DERL1 in SPC-A1 cells. GAPDH served as an internal control.

598 resulted in distinctly increased metastatic nodes as compared to the control group. Furthermore, we investigated the metastasizing node by IHC and H\&E staining as presented in Figs. 6B and 6C. In summary, these results indicated that miR-598 inhibits tumor metastasis of NSCLC in vivo.

\section{Discussion}

Lung cancer is the most frequent cause of malignant tumor-related deaths [24] due to which, the five-year survival rate is $<20 \%$ owing to diagnosis at an advanced stage. Despite the advances in surgery and chemotherapy, patients with lung cancer continue to suffer from the side effects of chemotherapy and considerably high recurrence rate. Therefore, finding novel molecules or therapeutic targets that could suppress the invasion and migration of lung cancer is an urgent requisite.

In recent years, miRNAs have been demonstrated to play pivotal roles in tumorigenesis and metastasis of various cancers including lung cancer [25-27]. Presently, several studies have explored the role of miR-598 in the development of various cancers. The overexpression of miR-598 suppressed the proliferation, migration, and invasion of osteosarcoma cells [13]. Moreover, miR-598 always inhibits EMT and suppresses metastasis in colorectal cancer by targeting JAG1 [14]. However, the role of miR-598 in NSCLC has not been reported previously.

To the best of our knowledge, miRNAs are involved in the progression of cancers by directly regulating the expression of specific target genes. We identified DERL1 as a direct downstream target gene of miR-598 using bioinformatics method that was further confirmed by the luciferase reporter assay.
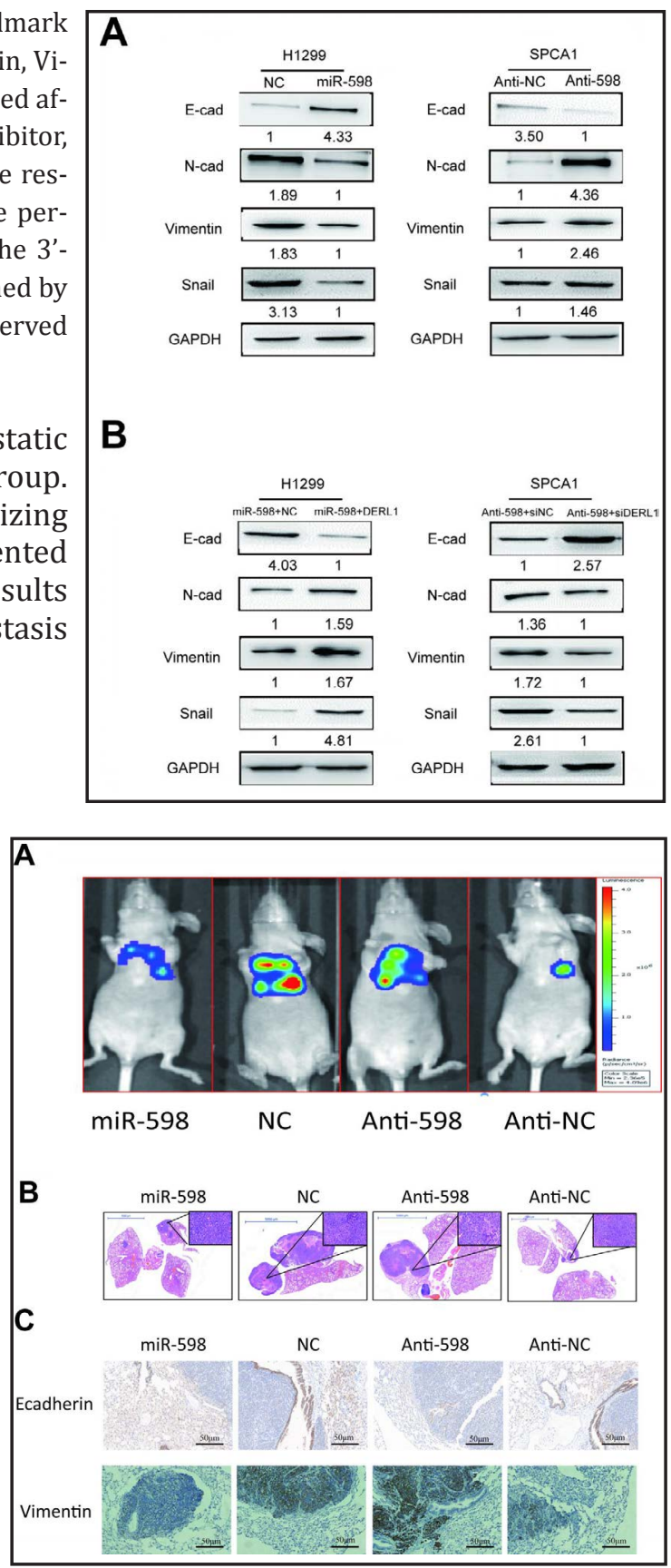

Fig. 6. MiR-598 inhibits NSCLC metastasis in vivo. A: The metastatic lung nodule was detected by IVIS@ Lumina II system. B: H\&E staining was used to identify the altered effects of miR-598 expression on cell metastasis in the samples collected from nude mice of lung tissues. C: IHC staining against E-cadherin and Vimentin assay determined the alteration on EMT hallmark genes in the samples collected from the lung tissues of nude mice. 
DERL1 is overexpressed in NSCLC; it also promotes cancer cell invasion via EGFR-ERKmediated upregulation of MMP-2 and MMP-9 $[16,19,21]$. However, the correlation between DERL1 and miR-598 remains unclear.

Herein, we implemented a series of experiments both in vitro and in vivo to investigate the role of miR-598 in NSCLC progression and found that miR-598 was downregulated in NSCLC cell lines and tissue specimens. Reportedly, A549 and H1299 are highly metastatic cell lines, while SPCA1 and H358 are weakly metastatic cell lines [23]. The current results showed that the expression level of miR-598 was markedly suppressed in A549 and H1299 as compared to SPCA1 and H358. This result also confirmed that miR-598 expression was related to cancer cell metastasis ability. The overexpression of miR-598 in NSCLC inhibits tumor cell invasion and migration in vitro. Furthermore, the in vivo animal experiments demonstrated that the overexpression of miR-598 could inhibit the tumor cell metastasis in NSCLC. Subsequently, miR-598 can rescue the stimulation effect of DERL1 on the invasion and migration of NSCLC cells. Based on the above results, for the first time, we demonstrated that DERL1 is a direct downstream target of miR-598.

In order to invade and metastasize, tumor cells would undergo a pivotal physiological process termed as EMT, which means that tumor epithelial cells gradually transform into mesenchymal-like cells by shedding their epithelial characteristics and acquiring those of mesenchyma [28-30]. Increasing evidence has suggested that miRNAs are responsible for the occurrence and metastasis of lung cancer involving the regulation of EMT [31-33]. IHC showed a positive correlation between the expressions of miR-598 and E-cadherin and a negative correlation between that of miR-598 and Vimentin. The in vitro experiment demonstrated that overexpression of miR-598 led to the increased expression of E-cadherin, while the expression of $\mathrm{N}$-cadherin, Vimentin, and Snail was decreased as assessed by Western blot. Depending on the rescue assay, the EMT markers are altered reversely in response to co-transfection with miR-598 mimics and pcDNA3.1-DERL1 in NSCLC cells. Thus, we speculated that the overexpression of miR-598 could inhibit invasion and migration via suppression of DERL1 and EMT in NSCLC.

In summary, the current study demonstrated miR-598 as a tumor suppressor by negatively regulating DERL1 and EMT to suppress the invasion and migration in NSCLC, thereby serving as a novel therapeutic target for the clinical treatment of NSCLC.

\section{Abbreviations}

NSCLC (non-small cell lung cancer); SCLC (small cell lung cancer); miRNA (microRNA); qRT-PCR (quantitative real-time polymerase chain reaction); EMT (epithelialmesenchymal transition); 3'-UTR (3'-untranslated region); FBS (fetal bovine serum); IHC (immunohistochemistry); H\&E (hematoxylin and eosin).

\section{Acknowledgements}

This work was supported by grants from the Natural Science Foundation of Jiangsu Province (Grants No BK20171484), the Project of Invigorating Health Care through Science, Technology, and Education (Jiangsu Provincial Medical Youth Talent QNRC2016856), the National Natural Science Foundation of China (No. 81672896), the Summit of the Six Top Talents Program of Jiangsu Province (2017-WSN-179), Postgraduate Research \& Practice Innovation Program of Jiangsu Province, and the Priority Academic Program Development of Jiangsu Higher Education Institutions (JX10231801).

\section{Disclosure Statement}

The authors declare to have no competing interests. 


\section{Cellular Physiology Cell Physiol Biochem 2018;47:245-256

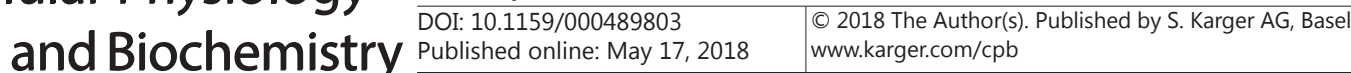

Yang et al.: Mir-598 in NSCLC

\section{References}

1 Siegel RL, Miller KD, Jemal A: Cancer Statistics, 2017 CA Cancer J Clin 2017;67:7-30.

2 Ramalingam SS, Owonikoko TK, Khuri FR: Lung Cancer: New Biological Insights and Recent Therapeutic Advances. CA Cancer J Clin 2011;61:91-112.

3 Filaire E, Dupuis C, Galvaing G, Aubreton S, Laurent H, Richard R, Filaire M: Lung cancer: What are the links with oxidative stress, physical activity and nutrition. Lung Cancer 2013;82:383-389.

4 Aisner DL, Marshall CB: Molecular Pathology of Non-Small Cell Lung Cancer A Practical Guide. Am J Clin Pathol 2012;138:332-346.

5 Zhou YF, Li SX, Li JT, Wan DF, Li QX: Effect of microRNA-135a on Cell Proliferation, Migration, Invasion, Apoptosis and Tumor Angiogenesis Through the IGF-1/PI3K/Akt Signaling Pathway in Non-Small Cell Lung Cancer. Cell Physiol Biochem 2017;42:1431-1446.

-6 Ribeiro AO SC, Izzotti A, Pereira LV, Vasques LR: MicroRNAs: Modulators of Cell Identity, and their Applications in Tissue Engineering. MicroRNA 2014;3:45-53.

7 Yang FM, Ning ZQ, Ma L, Liu WT, Shao CC, Shu YQ, Shen H: Exosomal miRNAs and miRNA dysregulation in cancer-associated fibroblasts. Mol Cancer 2017;16:148.

8 Lin L, Tu HB, Wu L, Liu M, Jiang GN: MicroRNA-21 Regulates Non-Small Cell Lung Cancer Cell Invasion and Chemo-Sensitivity through SMAD7. Cell Physiol Biochem 2016;38:2152-2162.

-9 Chen X, Ba Y, Ma LJ, Cai X, Yin Y, Wang KH, Guo JG, Zhang YJ, Chen JN, Guo X, Li QB, Li XY, Wang WJ, Zhang Y, Wang J, Jiang XY, Xiang Y, Xu C, Zheng PP, Zhang JB, Li RQ, Zhang HJ, Shang XB, Gong T, Ning G, Wang J, Zen $\mathrm{K}$, Zhang JF, Zhang CY: Characterization of microRNAs in serum: a novel class of biomarkers for diagnosis of cancer and other diseases. Cell Res 2008;18:997-1006.

10 Lu J, Getz G, Miska EA, Alvarez-Saavedra E, Lamb J, Peck D, Sweet-Cordero A, Ebet BL, Mak RH, Ferrando AA, Downing JR, Jacks T, Horvitz HR, Golub TR: MicroRNA expression profiles classify human cancers. Nature 2005;435:834-838.

11 Varambally S, Cao Q, Mani RS, Shankar S, Wang XS, Ateeq B, Laxman B, Cao XH, Jing XJ, Ramnarayanan K, Brenner JC, Yu JD, Kim JH, Han B, Tan P, Kumar-Sinha C, Lonigro RJ, Palanisamy N, Maher CA, Chinnaiyan AM: Genomic Loss of microRNA-101 Leads to Overexpression of Histone Methyltransferase EZH2 in Cancer. Science 2008;322:1695-1699.

12 Chen Y, Gao YP, Zhang K, Li C, Pan Y, Chen J, Wang R, Chen LB: MicroRNAs as Regulators of Cisplatin Resistance in Lung Cancer. Cell Physiol Biochem 2015;37:1869-1880.

13 Liu K, Sun XL, Zhang YG, Liu L, Yuan QL: MiR-598: A tumor suppressor with biomarker significance in osteosarcoma. Life Sci 2017;188:141-148.

14 Chen J, Zhange HC, Chen Y, Qiao GL, Jiang WH, Ni PH, Liu XF, Ma LJ: miR-598 inhibits metastasis in colorectal cancer by suppressing JAG1/Notch2 pathway stimulating EMT. Exp Cell Res 2017;352:104-112.

15 Liang CJ, Chang YC, Chang HC, Wang CK, Hung YC, Lin YE, Chan CC, Chen CH, Chang HY, Sang TK: Derlin-1 Regulates Mutant VCP-Linked Pathogenesis and Endoplasmic Reticulum Stress-Induced Apoptosis. Plos Genetics 2014;10: e1004675.

16 Ran Y, Hu H, Hu D, Zhou Z, Sun Y, Yu L, Sun L, Pan J, Liu J, Liu T, Yang Z: Derlin-1 is overexpressed on the tumor cell surface and enables antibody-mediated tumor targeting therapy. Clin Cancer Res 2008;14:65386545.

17 Wang F, Olson EM, Shyng SL: Role of Derlin-1 Protein in Proteostasis Regulation of ATP-sensitive Potassium Channels. J Biol Chem 2012;287:10482-10493.

18 Li DJ, Shi M, Ji HS, Chen G, Jiang H, Wang Z: MicroRNA-181d is a tumor suppressor in human esophageal squamous cell carcinoma inversely regulating Derlin-1. Oncol Rep 2016;36:2041-2048.

19 Tan XM, He XL, Jiang ZH, Wang XH, Ma LM, Liu L, Wang X, Fan ZN, Su DM: Derlin-1 is overexpressed in human colon cancer and promotes cancer cell proliferation. Mol Cell Biochem 2015;408:205-213.

20 Wu ZY, Wang C, Zhang Z, Liu WL, Xu HS, Wang HQ, Wang Y, Zhang W, Wang SL: High Expression of Derlin-1 Is Associated with the Malignancy of Bladder Cancer in a Chinese Han Population. PLoS One 2016;11: e0168351.

21 Dong QZ, Wang Y, Tang ZP, Fu L, Li QC, Wang ED, Wang EH: Derlin-1 Is Overexpressed in Non-Small Cell Lung Cancer and Promotes Cancer Cell Invasion via EGFR-ERK-Mediated Up-Regulation of MMP-2 and MMP-9. Am J Pathol 2013;182:954-964. 


\section{Cellular Physiology Cell Physiol Biochem 2018;47:245-256 \begin{tabular}{l|l} 
DOI: 10.1159/000489803 & and Biochemistry \\
Published online: May 17, 2018 & $\begin{array}{l}\text { O } 2018 \text { The Author(s). Published by S. Karger AG, Basel } \\
\text { www.karger.com/cpb }\end{array}$
\end{tabular}}

Yang et al.: Mir-598 in NSCLC

22 Xu L, Wang ZH, Xu D, Lin G, Li DR, Wan T, Guo SL: Expression of Derlin-1 and its effect on expression of autophagy marker genes under endoplasmic reticulum stress in lung cancer cells. Cancer Cell Int 2014;14:50.

23 Yu T, Li J, Yan M, Liu L, Lin H, Zhao F, Sun L, Zhang Y, Cui Y, Zhang F, Li J, He X, Yao M: MicroRNA-193a-3p and -5p suppress the metastasis of human non-small-cell lung cancer by downregulating the ERBB4/PIK3R3/ mTOR/S6K2 signaling pathway. Oncogene 2015;34:413-423.

-24 Wong MCS, Lao XQ, Ho KF, Goggins WB, Tse SLA: Incidence and mortality of lung cancer: global trends and association with socioeconomic status. Sci Rep 2017;7:14300.

25 Yang X, Chen YT, Chen LB: The Versatile Role of microRNA-30a in Human Cancer. Cell Physiol Biochem 2017;41:1616-1632.

-26 Chan B, Manley J, Lee J, Singh SR: The emerging roles of microRNAs in cancer metabolism. Cancer Lett 2015;356:301-308.

27 Lujambio A, Lowe SW: The microcosmos of cancer. Nature 2012;482:347-355.

28 Thiery JP: Epithelial-mesenchymal transitions in tumour progression. Nature Reviews Cancer 2002;2:442454.

29 Zhou PT, Li B, Liu FR, Zhang MC, Wang Q, Liu YH, Yao Y, Li D: The epithelial to mesenchymal transition (EMT) and cancer stem cells: implication for treatment resistance in pancreatic cancer. Mol Cancer 2017;16:52.

-30 Hu QF, Tong SJ, Zhao XJ, Ding WH, Gou YC, Xu K, Sun CY, Xia GW: Periostin Mediates TGF-beta-Induced Epithelial Mesenchymal Transition in Prostate Cancer Cells. Cell Physiol Biochem 2015;36:799-809.

-31 Koutsaki M, Spandidos DA, Zaravinos A: Epithelial-mesenchymal transition-associated miRNAs in ovarian carcinoma, with highlight on the miR-200 family: Prognostic value and prospective role in ovarian cancer therapeutics. Cancer Lett 2014;351:173-181.

-32 Xiang XJ, Deng J, Liu YW, Wan LY, Feng M, Chen J, Xiong JP: MiR-1271 Inhibits Cell Proliferation, Invasion and EMT in Gastric Cancer by Targeting FOXQ1. Cell Physiol Biochem 2015;36:1382-1394.

-33 Ying XY, Wei K, Lin Z, Cui YG, Ding J, Chen Y, Xu BQ: MicroRNA-125b Suppresses Ovarian Cancer Progression via Suppression of the Epithelial-Mesenchymal Transition Pathway by Targeting the SET Protein. Cell Physiol Biochem 2016;39:501-510. 